\title{
DIFFERENTIABLE SEMIGROUPS WITH A NEIGHBORHOOD OF THE IDENTITY HOMEOMORPHIC TO A BANACH SPACE
}

\author{
J. W. NEUBERGER ${ }^{1}$
}

\begin{abstract}
Suppose $S$ is a topological semigroup with identity $e, H$ is a Banach space and there is a homeomorphism $g$ from an open subset of $S$ containing $e$ onto $H$. Theorem. Suppose that $V(x, y)=g\left(g^{-1}(x) g^{-1}(y)\right)$ for all $x, y$ in $H$ for which the second expression is defined. If $V$ is $C^{(2)}$ then there is an open subset $D$ of $S$ containing $e$ so that if $q$ is in $D$, then for some number $t$ and some one-parameter subgroup $f$ of $S, q=f(t)$.
\end{abstract}

Suppose $S$ is a topological semigroup with identity $e$ and $H$ is a Banach space. Suppose furthermore that there is a homeomorphism $g$ from an open subset of $S$ containing $e$ onto $H$ so that $g(e)=0$. Denote by $V$ the function with domain all $(x, y)$ in $H \times H$ for which $g^{-1}(x) g^{-1}(y)$ is in $D(g)$ so that $V(x, y)=g\left(g^{-1}(x) g^{-1}(y)\right)$ for all such $(x, y)$. A member $(x, y)$ of $H \times H$ is sometimes denoted by $\left(\begin{array}{l}x \\ y\end{array}\right)$ and $\|(x, y)\|$ is defined to be

$$
\max (\|x\|,\|y\|) \text {. }
$$

A one-parameter subgroup of $S$ is a continuous function $f$ from the real numbers $R$ to $S$ such that $f(0)=e$ and $f(r) f(s)=f(r+s)$ for all $r, s$ in $R$. Clearly the range of such a function $f$ is a subgroup of $S$.

The main object of this note is to prove the following:

THEOREM. If $V$ is $C^{(2)}$ (twice continuously differentiable in the sense of Fréchet ([2, VIII.1] or [7, II.1.2])) then there is an open subset $D$ of $S$ containing $e$ so that if $q$ is in $D$, then for some number $t$ and some oneparameter subgroup $f$ of $S, q=f(t)$.

Before a proof of this theorem is stated some preliminary remarks and definitions are given.

This theorem is related to a theorem of Mostert and Shields [4] which gives a similar result (but without the differentiability condition) in the case $H$ is finite dimensional. Some of the ideas in the proof of the present

Received by the editors February 11, 1971.

AMS 1970 subject classifications. Primary 22A20.

Key words and phrases. Topological semigroup, differentiable, manifold.

${ }^{1}$ Supported in part by a National Science Foundation grant.

(c) American Mathematical Society 1972 
theorem can be found in [1] and [6]. The treatment of differentiable topological semigroups here parallels that of [3] for differentiable topological groups on a Banach manifold.

The writer is indebted to Alexander Doniphan Wallace for his suggestion (some years ago) that certain developments in [5] might be applicable to the study of topological semigroups.

Definition. If $F$ is a function from a subset of $R$ containing 0 to $S$ and $t$ is a number, then the statement that $F$ is product integrable from 0 to $t$ means that there is an element $p$ of $S$ so that if $U$ is an open set containing $p$, then there is $\delta>0$ so that if $t_{0}, t_{1}, \cdots, t_{n+1}$ is a partition of $[0, t]$ with mesh $<\delta$, then $F\left(t_{n+1}-t_{n}\right) \cdots F\left(t_{1}-t_{0}\right)$ is in $U$. Such a (necessarily unique) point is denoted by $\pi_{0}^{t} F$.

Definition. If $G$ is a function from a subset $R_{1}$ of $R$ containing 0 so that for each $t$ in $R_{1}, G(t)$ is a transformation from a subset of $H$ to $H$ and $p$ is in $H$, then the statement that $G$ is product integrable from 0 to $t$ at $p$ means that there is a point $q$ in $H$ so that if $\varepsilon>0$, then there is $\delta>0$ so that if $t_{0}, t_{1}, \cdots, t_{n+1}$ is a partition of $[0, t]$ with mesh $<\delta$, then

$$
\left\|G\left(t_{n+1}-t_{n}\right) \cdots G\left(t_{1}-t_{0}\right) p-q\right\|<\varepsilon .
$$

Such a (necessarily unique) point $q$ is denoted by $\pi_{0}^{t}(G, p)$.

Proof of Theorem. For $\varepsilon>0$ denote by $R(\varepsilon)$ the set of all $x$ in $H$ so that $\|x\| \leqq \varepsilon$. Denote by $\delta^{\prime}$ a positive number so that if $\|x\|,\|y\|<\delta^{\prime}$ then $(x, y)$ is in $D(V)$, i.e., $g^{-1}(x) g^{-1}(y)$ is in $D(g)$. If $c$ is in $R(1)$, denote $V^{\prime}(0, y)\left(\begin{array}{c}c \\ 0\end{array}\right)$ by $A_{c}(y)$ for all $y$ in $R(1)$. Since each of $V^{\prime}$ and $V^{\prime \prime}$ is continuous at $(0,0)$, there exist $\delta_{0}, M>0$ with $\delta_{0}<\delta^{\prime}$ such that $\left|V^{\prime}(x, y)\right| \leqq M$ and $\left|V^{\prime \prime}(x, y)\right| \leqq M$ if $\|x\|,\|y\|<\delta_{0}$ (the first norm is that for bounded linear transformations from $H \times H$ to $H$, the second is for bounded bilinear functions from $(H \times H) \times(H \times H)$ to $H$, i.e.,

$$
\left|V^{\prime \prime}(x, y)\right|=\inf \left\{k:\left\|\left(V^{\prime \prime}(x, y)\right)(p, q)\right\| \leqq k\|p\|\|q\|\right.
$$

for all $p$ and $q$ in $H \times H\})$.

If $c$ is in $R(1)$, then $G_{c}$ denotes the function with domain $\left(-\delta_{0}, \delta_{0}\right)$ so that if $|t|<\delta_{0}$, then $G_{c}(t)$ is the transformation from $R\left(\delta_{0}\right)$ to $H$ so that $\left(G_{c}(t)\right) y=V(t c, y)$ for all $y$ in $R\left(\delta_{0}\right)$. Denote $g^{-1}(t c)$ by $F_{c}(t)$ if $|t|<\delta_{0}$ and $c$ is in $R(1)$. A series of inequalities follow. Suppose $\|c\| \leqq 1$.

(1) If each of $x, y$ is in $R\left(\delta_{0}\right)$, then

$$
\begin{aligned}
\left\|A_{c}(x)-A_{c}(y)\right\| & =\left\|V^{\prime}(0, x)\left(\begin{array}{l}
c \\
0
\end{array}\right)-V^{\prime}(0, y)\left(\begin{array}{l}
c \\
0
\end{array}\right)\right\| \\
& =\left\|\int_{0}^{1} d s V^{\prime \prime}(0, y+s(x-y))\left(\left(\begin{array}{l}
c \\
0
\end{array}\right),\left(\begin{array}{c}
0 \\
x-y
\end{array}\right)\right)\right\| \\
& \leqq M\|x-y\| .
\end{aligned}
$$


(2) If $y$ is in $R\left(\delta_{0}\right)$ then

$$
\left\|A_{c}(y)\right\|=\left\|V^{\prime}(0, y)\left(\begin{array}{l}
c \\
0
\end{array}\right)\right\| \leqq M\|c\| \leqq M .
$$

(3) If $\|y\|<\delta_{0}$ and $|t|<\delta_{0}$, then

$$
\begin{aligned}
\left\|G_{c}(t) y-y\right\| & =\|V(t c, y)-V(0, y)\| \\
& =\left\|\int_{0}^{1} d s V^{\prime}(s t c, y)\left(\begin{array}{c}
t c \\
0
\end{array}\right)\right\| \leqq|t| M
\end{aligned}
$$

and so $G_{c}(t) y$ is in $R\left(\delta_{0}\right)$ if $|t| M+\|y\| \leqq \delta_{0}$.

(4) If each of $x, y$ is in $R\left(\delta_{0}\right)$ and $|t|<\delta_{0}$, then

$$
\begin{aligned}
\left\|G_{c}(t) x-G_{c}(t) y\right\|=\|V(t c, x)-V(t c, y)\| & \left\|\int_{0}^{1} d s V^{\prime}(t c, y+s(x-y))\left(\begin{array}{c}
0 \\
x-y
\end{array}\right)\right\| \\
\leqq & \| \int_{0}^{1} d s\left[V^{\prime}(t c, y+s(x-y))\left(\begin{array}{c}
0 \\
x-y
\end{array}\right)\right. \\
& \left.-V^{\prime}(0, y+s(x-y))\left(\begin{array}{c}
0 \\
x-y
\end{array}\right)\right] \| \\
= & \left.\| \int_{0}^{1} d s \int_{0}^{1} d r V^{\prime \prime}(-r t c, y+s(x-y))\left(\begin{array}{c}
-t c \\
0
\end{array}\right),\left(\begin{array}{c}
0 \\
x-y
\end{array}\right)\right) \| \\
& +\left\|\int_{0}^{1} d s V^{\prime}(0, y+s(x-y))\left(\begin{array}{c}
0 \\
x-y
\end{array}\right)\right\| \\
\leqq & M|t|\|c\|\|x-y\|+\|x-y\| \leqq(1+M|t|)\|x-y\|
\end{aligned}
$$

since $V^{\prime}(0, y+s(x-y))\left(\begin{array}{c}0 \\ x-y\end{array}\right)=\left(\begin{array}{c}0 \\ x-y\end{array}\right)$ for all $s$ in $[0,1]$.

Denote $\delta_{0} /(2 M)$ by $\delta$. One has (using (2) and Theorem 10.4.5 of [2], for example) that there is a unique function $Y$ from $[-2 \delta, 2 \delta]$ to $R\left(\delta_{0}\right)$ so that $Y(0)=0$ and $Y^{\prime}(t)=A_{c}(Y(t))$ if $|t| \leqq 2 \delta$. Note that $\|Y(t)\| \leqq|t| M$ if $|t| \leqq 2 \delta$.

Denote by $u$ the function from $[-2 \delta, 2 \delta] \times R(1)$ to $R\left(\delta_{0}\right)$ so that $u(0, c)=0$ and $u_{1}(t, c)=A_{c}(u(t, c))$ if $c$ is in $R(1)$ and $|t| \leqq 2 \delta\left(u_{1}(t, c)\right.$ is the partial derivative in the first place of $u$ at $(t, c)$-it is a member of $H$ ). It follows from [2, Theorem 10.7.3] that $u_{2}$ exists and is continuous on $(-2 \delta, 2 \delta) \times H_{0}, H_{0}$ being the open unit ball of $H$. (For $|t|<2 \delta$ and $c$ in $H_{0}, u_{2}(t, c)$ is the partial derivative in the second place of $u$ at $(t, c)$-it is 
a bounded linear transformation from $H$ to $H$.) Moreover, using 10.7.3.1 of [2], it follows that $u_{1,2}(t, 0)=I$ if $|t|<2 \delta$ and so $u_{2}(t, 0)=t I$ if $|t|<2 \delta$. Hence, setting $E_{1}(c)=u(\delta / 2, c)$ for all $c$ in $H_{0}$, one has that $E_{1}^{\prime}$ is continuous on $H_{0}$. Since $E_{1}^{\prime}(0)=(\delta / 2) I$ (and hence $E_{1}^{\prime}(0)$ has a bounded inverse which is from $H$ to $H$ ) there is an open subset $D_{1}^{\prime}$ of $R(1)$ [2, Theorem 10.2.5] containing 0 and an open set $D_{2}^{\prime}$ containing 0 so that the restriction to $D_{1}^{\prime}$ of $E_{1}$ is a homeomorphism from $D_{1}^{\prime}$ onto $D_{1}^{\prime}$. Denote by $\delta_{1}$ a positive number $\leqq \delta_{0} / 2$ so that if $\|x\|<\delta_{1}$ then $x$ is in $D_{2}^{\prime}$. Denote by $E$ the restriction of $E_{1}$ whose range is $D_{1}=\left\{x \in H ;\|x\|<\delta_{1}\right\}$.

(5) If $c$ is in $R(1),|t|<2 \delta,|t+\Delta|<2 \delta$ and $|\Delta|<\delta_{0}$, then

$$
\begin{aligned}
& \left\|G_{c}(\Delta) u(t, c)-u(t+\Delta, c)\right\| \\
& =\left\|V(\Delta c, u(t, c))-\left(u(t, c)+\int_{t}^{t+\Delta} d s A_{c}(u(s, c))\right)\right\| \\
& =\left\|V(\Delta c, U(t, c))-V(0, u(t, c))-\int_{t}^{t+\Delta} d s V^{\prime}(0, u(s, c))\left(\begin{array}{l}
c \\
0
\end{array}\right)\right\| \\
& =\left\|\int_{t}^{t+\Delta} d s\left[V^{\prime}((s-t) c, u(t, c))\left(\begin{array}{l}
c \\
0
\end{array}\right)-V^{\prime}(0, u(s, c))\left(\begin{array}{l}
c \\
0
\end{array}\right)\right]\right\| \\
& =\| \int_{t}^{t+\Delta} d s \int_{0}^{1} d r V^{\prime \prime}((1-r)(s-t) c, u(t, c)+r(u(s, c)-u(t, c))) \\
& \leqq|\Delta|^{2} M^{2} \quad \cdot\left(\left(\begin{array}{l}
c \\
0
\end{array}\right),\left(\begin{array}{c}
-(s-t) c \\
u(s, c)-u(t, c)
\end{array}\right)\right) \|
\end{aligned}
$$

since $\|u(s, c)-u(t, c)\| \leqq M|s-t|$ if $|s|,|t| \leqq 2 \delta$ and $\|c\| \leqq 1$.

Now suppose $|s|,|t| \leqq \delta / 2, s_{0}, s_{1}, \cdots, s_{m+1}$ is a partition from $t$ to $s$ and $a_{i}=s_{i+1}-s_{i}, i=0,1, \cdots, m$. Then, using (3) repeatedly,

$$
\begin{aligned}
\| G_{c}\left(a_{i}\right) \cdots & G_{c}\left(a_{0}\right) u(t, c)-u(t, c) \| \\
& \leqq\left(\left|a_{i}\right|+\cdots+\left|a_{0}\right|\right) M=\left|s_{i+1}-t\right| M \leqq \delta M=\delta_{0} / 2
\end{aligned}
$$

so that $G_{c}\left(a_{i}\right) \cdots G_{c}\left(a_{0}\right) u(t, c)$ is in $R\left(3 \delta_{0} / 4\right), i=0,1, \cdots, m$. Similarly,

$$
\begin{aligned}
\| G_{c}\left(a_{j}\right) \cdots & G_{c}\left(a_{i}\right) u\left(s_{i}, c\right)-u\left(s_{i}, c\right) \| \\
& \leqq \\
& \left(\left|a_{j}\right|+\cdots+\left|a_{i}\right|\right) M \leqq\left|s_{j+1}-s_{i}\right| M
\end{aligned}
$$

and hence

$$
\begin{aligned}
\left\|G_{c}\left(a_{j}\right) \cdots G_{c}\left(a_{i}\right) u\left(s_{i}, c\right)\right\| & \leqq\left|s_{j+1}-s_{i}\right| M+\left\|u\left(s_{i}, c\right)\right\| \\
& \leqq\left|s_{j+1}-s_{i}\right| M+\left|s_{i}\right| M \\
& \leqq \delta M+(\delta / 2) m=3 \delta_{0} / 4 \quad \text { if } m \geqq j \geqq i .
\end{aligned}
$$


Hence

$$
\begin{aligned}
\| G_{c}\left(a_{m}\right) \cdots & G_{c}\left(a_{0}\right) u(t, c)-u(s, c) \| \\
& \times \sum_{i=0}^{m}\left\|G_{c}\left(a_{m}\right) \cdots G_{c}\left(a_{i}\right) u\left(s_{i}, c\right)-G_{c}\left(a_{m}\right) \cdots G_{c}\left(a_{i+1}\right) u\left(s_{i+1}, c\right)\right\| \\
\leqq & \sum_{i=0}^{m}\left(1+\left|a_{m}\right| M\right) \cdots\left(1+\left|a_{i+1}\right| M\right)\left\|G_{c}\left(a_{i}\right) u\left(s_{i}, c\right)-u\left(s_{i+1}, c\right)\right\| \\
\leqq & (\exp |s-t|) \sum_{i=0}^{m} M^{2}\left|a_{i}\right|^{2} \\
& \leqq \\
& M^{2}(\exp |s-t|)|s-t| \operatorname{mesh}\left(s_{0}, s_{1}, \cdots, s_{m+1}\right),
\end{aligned}
$$

using (4) repeatedly and (5) together with the fact that $1+\varepsilon \leqq \exp \varepsilon$ if $\varepsilon \geqq 0$. The product integrability of $G_{c}$ from 0 to $s-t$ at $u(t, c)$ follows. Clearly

$$
\pi_{0}^{s-t}\left(G_{c}, u(t, c)\right)=u(s, c) .
$$

Note that for $s, t, s_{0}, s_{1}, \cdots, s_{m+1}, a_{0}, a_{1}, \cdots, a_{m}$ as in the preceding paragraph it follows that

$$
g^{-1}\left(G_{c}\left(a_{m}\right) \cdots G_{c}\left(a_{0}\right) u(t, c)\right)=F_{c}\left(a_{m}\right) \cdots F_{c}\left(a_{0}\right) g^{-1}(u(t, c))
$$

Hence the product integrability of $G_{c}$ from 0 to $s$ at 0 implies the product integrability of $F_{c}$ from 0 to $s$. Moreover, $g^{-1}\left(\pi_{0}^{s}\left(G_{c}, 0\right)\right)=\pi_{0}^{s} F_{c}$. Finally if $|s|,|t| \leqq \delta / 2$ then $g^{-1}\left(\pi_{0}^{s-t}\left(G_{c}, u(t, c)\right)\right)=\left(\pi_{0}^{s-t} F_{c}\right) g^{-1}(u(t, c))$.

Recall $E$ is a homeomorphism from an open subset of $H$ containing 0 onto $D_{1}$. Note that $g^{-1}\left(D_{1}\right)$ is an open subset of $S$ containing $e$. Suppose $q$ is in $g^{-1}\left(D_{1}\right)$. Then $x=g(q)$ is in $D_{1}$ and hence $x$ is in $R\left(\delta_{0} / 2\right)$ and $x=E(c)$ for some $c$ in $R(1)$, i.e., $x=u(\delta / 2, c)$. Define

$$
f(t)=g^{-1}(u(t, c))=g^{-1}\left[\pi_{0}^{t}\left(G_{c}, 0\right)\right]=\pi_{0}^{t} F_{c}
$$

for $|t| \leqq \delta / 2$ and note that $f$ is continuous. It follows from $\left(^{*}\right)$ that

$$
u(s, c)=\pi_{0}^{s-t}\left(G_{c}, u(t, c)\right) \quad \text { if }|s|,|t| \leqq \delta / 2 .
$$

Hence if $|t|,|s| \leqq \delta / 2$ and $s-t=r$, then

$$
\begin{aligned}
f(s) & =g^{-1}(u(s, c))=g^{-1}\left[\pi_{0}^{r}\left(G_{c}, u(t, c)\right)\right] \\
& =\left(\pi_{0}^{r} F_{c}\right) g^{-1}(u(t, c))=\left(\pi_{0}^{r} F_{c}\right)\left(\pi_{0}^{t} F_{c}\right)=f(r) f(t) .
\end{aligned}
$$

So $f$ is a local one-parameter group. Since $f$ is continuous it can be extended to a one-parameter subgroup of $S$. Since

$$
f(\delta / 2)=g^{-1}(u(\delta / 2, c))=g^{-1}(x)=q
$$

the proof is completed. 
ADDED IN PROOF. It is observed that the theorem follows from developments in [1]. The present argument seems substantially different, however.

\section{REFERENCES}

1. Garrett Birkhoff, Analytical groups, Trans. Amer. Math. Soc. 43 (1938), 61-101.

2. J. Dieudonné, Foundations of modern analysis, Pure and Appl. Math., vol. 10, Academic Press, New York, 1960. MR 22 \#11074.

3. B. Maissen, Lie-Gruppen mit Banachräume als Parameterräume, Acta Math. 108 (1962), 229-270. MR 26 \#262.

4. P. S. Mostert and A. L. Shields, Semigroups with identity on a manifold, Trans. Amer. Math. Soc. 91 (1959), 380-389. MR 21 \#4204.

5. J. W. Neuberger, Continuous products and nonlinear integral equations, Pacific J. Math. 8 (1958), 529-549. MR 21 \#1509.

6. J. von Neumann, Über die analytischen Eigenschaften von Gruppen linearer Transformationen und ihrer Darstellungen, Math. Z. 30 (1929), 3-42.

7. F. Nevanlinna and R. Nevanlinna, Absolute analysis, Die Grundlehren der math. Wissenschaften, in Einzeldarstellungen mit besonderer Beruchsichtigung der Anwendungsgebiete, Band 102, Springer-Verlag, Berlin, 1959. MR 22 \#12176.

Department of Mathematics, Emory University, Atlanta, Georgia 30322 